\title{
Liste des exercices
}

\section{Chapitre premier : pages $54-58$}

1. Fonctions de réponse associées à des opérateurs linéaires

2. Fonction de réponse d'un circuit RLC

3. Particule brownienne chargée

4. Raie d'absorption

5. Application des relations de Kramers-Kronig en astrophysique

6. Règles de somme

7. Réponse à un bruit

8. Relations de Kramers-Kronig pour un métal

9. Propagation des signaux dans les milieux diélectriques

\section{Chapitre 2 : pages 128-141}

1. Fonction de Green $G_{\infty}$ du Laplacien en 3d

2. Fonction de Green $G_{\infty}$ du Laplacien en dimension $d \geq 3$

3. Fonctions de Green du Laplacien en $1 \mathrm{~d}$ et $2 \mathrm{~d}$

4. Symétrie des fonctions de Green du Laplacien avec C.L. de Dirichlet homogènes

5. Fonctions de Green de Neumann spéciales du Laplacien

6. Règles de somme et résolvante

7. Plan conducteur

8. Fonctions de Green du Laplacien en coordonnées sphériques

9. Charge ponctuelle dans une sphère conductrice

10. Charge ponctuelle et sphère diélectrique

11. Fonction de Green $G_{\infty}$ du Laplacien en coordonnées cylindriques

12. Tenseur d'Oseen

13. Fonction de Green en théorie de l'élasticité

14. Laplacien discret et réseau de résistances

15. Méthode des images pour un problème bidimensionnel 
16. Hangar semi-cylindrique soumis au vent

17. Opérateur de Dirac

18. Avance du périhélie de Mercure

19. Oscillateur harmonique en présence d'une impureté

\section{Chapitre 3 : pages 236-242}

1. Unicité des solutions des équations de diffusion et de d'Alembert

2. Relations de réciprocité

3. Équation pour les câbles longs

4. Conditions de Neumann en théorie de la diffraction

5. Fonction de Green du d'Alembertien en dimension $2+1$

6. Fonction de Green du d'Alembertien en dimension $1+1$

7. Fonction de Green $G_{\infty}$ du Laplacien en dimension $d \geq 3$

8. Diffusion de la chaleur dans une boule

9. Des conditions de Dirichlet aux conditions de Robin

10. Conditions de Robin pour l'équation de la chaleur

11. Équation de Cattaneo en $3 d$

12. Équation de Klein-Gordon

\section{Chapitre 4 : pages 294-298}

1. Comportement asymptotique de la fonction de Bessel $J_{0}$

2. Coefficients du binôme

3. Forme aymptotique de la fonction de Green de Helmholtz

4. Ensemble isotherme-isobare

5. Évolution d'un paquet d'ondes et vitesse de groupe

6. De la fonction de Green de Cattaneo à celle de l'équation de diffusion

7. Modèle d'Ising avec des interactions à longue portée

8. Marche aléatoire de Bernoulli

9. Oscillateur harmonique et théorie des nombres 